\title{
Health Care Worker's Experience on Rapid Diagnostic Test (Rdt) for Malaria and Their Views on Community Acceptability of Rdt in Rubavu District, Rwanda: Qualitative Study
}

\author{
Gashegu $\mathbf{M}^{1 *}$, Habtu $\mathbf{M}^{1}$, Kansiime $C^{2}$, Mochama $\mathbf{M}^{1}$ and Asiimwe $\mathbf{B}^{1,2}$ \\ ${ }^{1}$ Department of Public Health, School of Health Sciences, Mount Kenya University, Kigali Campus, Rwanda \\ ${ }^{2}$ Department of Microbiology and School of Public Health, College of Health Sciences, Makerere University, Mulago Hospital Complex, Kampala, Uganda
}

\begin{abstract}
Background: Malaria remains one of the major public health problems in Rwanda. The country introduced artemisinin combination therapy (ACT) at community level using community health workers (CHWs) for treatment of uncomplicated malaria in 2008. Use of ACT with reliance on presumptive diagnosis may lead to excessive use, increased costs and rise of drug resistance. Thus to overcome these challenges, rapid diagnostic tests (RDTs) was introduced to be done by CHWs. This study assessed health care workers experience regarding RDT and their feelings on acceptability of RDTs by community members.
\end{abstract}

Methods: This was a qualitative research approach conducted in five sectors of Rubavu District. Three focus group discussions (FGDs) among community health workers and five key informant interviews (KIls) were conducted among three in-charges of CHWs, supervisor of the in-charges of CHWs and district health officer. Data were transcribed and coded and then analysed thematically.

Results: The study found that CHWs were well experienced in using RDTs. Moreover, district community health supervisor and a district health director confirmed that $\mathrm{CHWs}$ were quite experienced in the services they provide. The study further indicated that community acceptability of RDTs for malaria testing was high from the views of health care workers compared to microscopy testing.

Conclusion: The data indicated that community health workers are well experienced in the use of RDT for malaria diagnosis and the views of CHWs indicate high acceptability of RDTs for malaria by the community.

Keywords: Health care worker; Experience; Views; Community; Acceptability; Malaria; Rapid diagnostic test

\section{Introduction}

The advantages of malaria rapid diagnostic test (RDT) in averting death among community members were well documented $[1,2]$ and is internationally acknowledged. World Health Organization (WHO) has endorsed RDTs as adjunct tests to microscopy for parasitological confirmation of malaria in routine fever case management at lower levels of healthcare [2]. However, in low income countries endorsing a new or improved health technology in itself does not guarantee enduser utilization [3]. Other studies conducted in developed nations reported several factors responsible for acceptance and use of new health technologies $[4,5]$. These factors include organizational features such as how well the new technology is integrated with existing technologies, workflow, and top management commitment to the new technology. Also cited are individual factors such as perceptions of negative effects on users, resistance to change, lack of control, and readiness for change, as well as job factors such as self-efficacy, level of education, previous experience with similar technology, age, gender, clarity on the reasons for the new technology, training, and participation.

In 2009, Rwanda achieved universal treatment of malaria cases using Artemisinine Combination Treatment (ACTs) both at health facility level and at community level using Community Health Workers (CHWs) who were trained to test malaria with Rapid Diagnostic Test (RDTs) [6]. To date more than 94 per cent of all sick children are treated within 24 hours and 99 per cent of malaria cases are laboratory diagnosed before receiving the correct treatment [6]. Currently, the malaria burden in Rwanda has transitioned from a nationwide problem into a focal problem mostly in six high malaria burden districts along the border in the Eastern (Bugesera, Gatsibo, Nyagatare and Kirehe) and Southern provinces (Huye and Nyaruguru), which account for over 60 per cent of the malaria burden [6].

In order to have effective case management of malaria, clinical assessment and laboratory confirmation through light microscopy or RDTs should be addressed prior to treatment with an effective antimalarial [7-10]. There is evidence that using artemisinin combination therapies (ACTs) at the community level using trained community health workers is feasible, effective, and saves lives [11,12].

The study by Mukanga et al. [13] shows that RDT acceptability among community members depend on their education level and health experience. While there is no study done in Rubavu assessing acceptability of the community of RDTs for malaria diagnosis, this study assessed acceptability of RDTs among community members and health care worker's experience regarding use of RDTs in Rubavu district, Rwanda.

*Corresponding author: Gashegu Misbah, Department of Public Health, Schoo of Health Sciences, Mount Kenya University, P.O. Box 5826, Kigali Campus Rwanda, Tel: (+205) 783343552 or 783895127; E-mail: misbahdjuma50@yahoo.fr

Received August 09, 2017; Accepted September 01, 2017; Published September 26, 2017

Citation: Gashegu M, Habtu M, Kansiime C, Mochama M, Asiimwe B (2017) Health Care Worker's Experience on Rapid Diagnostic Test (Rdt) for Malaria and Their Views on Community Acceptability of Rdt in Rubavu District, Rwanda: Qualitative Study. Malar Chemoth Cont Elimination 6: 157. doi: 10.4172/ 2470-6965.1000157

Copyright: (c) 2017 Gashegu M, et al. This is an open-access article distributed under the terms of the Creative Commons Attribution License, which permits unrestricted use, distribution, and reproduction in any medium, provided the original author and source are credited. 
Citation: Gashegu M, Habtu M, Kansiime C, Mochama M, Asiimwe B (2017) Health Care Worker's Experience on Rapid Diagnostic Test (Rdt) for Malaria and Their Views on Community Acceptability of Rdt in Rubavu District, Rwanda: Qualitative Study. Malar Chemoth Cont Elimination 6: 157. doi: 10.4172/ 2470-6965.1000157

Page 2 of 6

\section{Materials and Methods}

\section{Study design and population}

This study was carried out in Rubavu District, Rwanda. The study consisted of three focus group discussions (FGDs) among community health workers (CHWs). For FGDs eight people were selected in each focus group discussion. Since there were three focus group discussions, twenty four participants were selected. Five key informant interviews including three in-charges of CHWs, supervisor of the in-charges of CHWs at hospital level and District Health Officer. All participants aged between 22 and 64 years were selected purposively.

\section{Data collection methods}

A guide was developed for both FGDs and KII in English then translated into a local dialect 'Kinyarwanda'. The discussions were held in each health center of the study area at locations that ensured privacy and the comfort of participants. The principal investigator facilitated the discussions while a trained interviewer took notes. The discussions were also recorded with tape recorder.

\section{Data analysis}

Participants' responses were grouped and categorized manually. The unit of analysis was the transcripts from FGDs and KIIs. Recorded FGDs and KIIs data were transcribed and translated from Kinyarwanda to English. Transcripts were first read thorough several times while making notes on them. All transcripts were cross-checked to ensure completeness of data. Content analysis using both latent and manifest analysis was used. Data were condensed without losing quality as described above. All transcripts were read though and codes generated. Responses with similar codes were re-categorized under a unifying theme. A matrix was created and individual matrices were reviewed until an agreement is reached. The categories were then interpreted for their descriptive meaning. Descriptive quotes representing key themes were identified.

\section{Ethical approval}

Approval to carry out the study was sought and obtained from Mount Kenya University Rwanda, Rubavu district administration. Ethical clearance was obtained from ethical committee of Rubavu District Hospital. Written consent was obtained from all study participants after a detailed explanation of the purpose of the study before participating. All data and study subjects were assured of confidentiality and data safety.

\section{Result and Discussion}

\section{Community acceptability of rapid diagnostic test for malaria}

Majority of the participants from FGDs indicate that RDT for malaria is more acceptable than microscopy among community members of Rubavu district. This is explained by the following quotes which indicated high acceptability of RDT for malaria by the community:

"Patients are able to see the results because we (CHWs) show them and explain how the test works and then get the result in a short period of time. People admire it very much and we usually treat children and most of the time adults request that services to be provided to them" (FGD 1\&2 with CHWs) (Table 1).

"People appreciate the way we help them. For instance, one day

\begin{tabular}{|c|c|c|c|}
\hline Gender & Age categories & Marital status & Number \\
\hline Female & $40-50$ & Married & $3 \times 3$ \\
\hline \multirow[t]{2}{*}{ Male } & $20-30$ & Single & $\mathrm{T}$ \\
\hline & $40-50$ & Married & \\
\hline Total & & & 24 \\
\hline
\end{tabular}

during the night, there was a mother who came to my home to seek for treatment of her kid. I treated that kid very well and in the morning a mother back to me and said: "I have seen my child playing with others. So, go ahead!" Then she asked me: "When will you start treating us? I mean adults." She went on saying:"I don't have any problem, I have given medicines to my child and now I'm going to work." Really RDT test is the best way that all people appreciate very much. They keep on saying to us:"you promised to provide medical care for adults and yet you are helping children only. So, we need this service, please" (FGD $2 \& 3$ with CHWs).

"I trust that RDT is a good test that people admire and this make adult's people to request this service be provided to them because we treat them on time and in a short period of time. This system comes in need and in a good time I'm telling you. People believe the work done by $\mathrm{CHW}$ because in case of sickness they contact CHW for treatment of their children instead of looking for other services. When the results are positive, they accept medicine provided to them. RDT results are the same as the one of microscopy test. This test works well and we have confidence for it and also truly, we do our work very well and people trust RDT services that make them be confident for us"(FGD 2 with CHWs).

"People appreciate the way we help them. For instance, one day during the night, there was a mother who came to my home to seek for treatment of her kid. I treated that kid very well and in the morning a mother back to me and said: "I have seen my child playing with others. So, go ahead!". Then she asked me: "When will you start treating us? I mean adults." She went on saying:"I don't have any problem, I have given medicines to my child and now I'm going to work."Really RDT test is the best way that all people appreciate very much. They keep on saying to us:"you promised to provide medical care for adults and yet you are helping children only. So, we need this service, please" (FGD 3 with CHWs).

"RDTs for malaria diagnosis started at the right time, local population likes it so much because are very fast. Another thing I can share with you is that no parent now who may provide medicines to children without CHW intervention. There was a mother who came to me for her child health care and I asked her if she has provided any medicines to child and said: "No, I refused to give my child any medicines before your test confirmation." This shows how they believe RDT very much. As soon as you tell parents RDT result showing that the child has malaria, they feel satisfied and then when provide them malaria medicines, they back home happily. Community believes and highly appreciates RDTs for malaria done by CHWs" (FGD 3 with CHWs).

"People prefer RDT done by CHWs because the services of Health centers make people waiting for long period of time. People admire RDT. For example here, when we have many cases of malaria, people go to health center but because the results of microscopy are not quick, people claim for RDT because they know it and its efficacy. People like 
Citation: Gashegu M, Habtu M, Kansiime C, Mochama M, Asiimwe B (2017) Health Care Worker's Experience on Rapid Diagnostic Test (Rdt) for Malaria and Their Views on Community Acceptability of Rdt in Rubavu District, Rwanda: Qualitative Study. Malar Chemoth Cont Elimination 6: 157. doi: 10.4172/ 2470-6965.1000157

it so much and said that it is a response of getting health services near them. People cannot suffer from malaria for a long period of time. Having one symptom they immediately consult a CHW. A health care worker is selected at village level by community, so they select CHW they want who is honest. In addition to this, both sexes are included in HCWs. The comparison between the situation before and after RDT use shows clearly the significant improvement and HCWs' achievements in malaria control" (KII 1\&2).

"People accept RDT very well. From the discussion we have done with community, I have found that they trust it so much. When a CHW test patients using RDT they said that it is good because they trust them. Really people prefer to go to CHWs for RDT when they are malaria suspected" (KII 3)

On the other hand the KIIs among district community health supervisor and district health director also indicate that community accepts RDT for malaria diagnosis as follows:

"Parents prefer to take their children to CHWs because they get medical care quickly and the children's life became better without any problem. People believe it so much because when the result of RDT for malaria is positive, CHWs cure them. So, they know that CHWs are fit for their job" (KII 4\&5).

The above quotations show that community accepts RDT services done by CHWs as they are confident about them. The study found that community health workers (CHWs) were trusted by their communities because of their voluntary services, ease of access, and perceived effectiveness of the anti-malarial drugs they use [14]. This is in line with the findings from a multi-country study which showed high community acceptability of CHWs management of sick children, with most children receiving treatment on the same day or within one day of onset of symptoms [15]. Similarly, Kilian et al. [16] in western Uganda, found high acceptability of pre-packaged antimalarial drugs in the hands of CHWs. In the present study, the high community acceptability of RDTs was partly due to the appreciation that CHWs would be able to offer treatment which was based on test results rather than just presence of symptoms and signs. While RDTs have the potential to greatly enhance the management of febrile illness the success of their use in the hands of CHWs is highly dependent on how the community will accept the services provided.

\section{Health care worker's experiences regarding use of RDTs for malaria}

Both FGDs and KIIs indicate that community health workers have high level of experience in the use of RDT for malaria diagnosis as shown below:

"It is one year and above after being trained. We are enough experienced and nobody can be mistaken about the result of malaria. All of us we are able to test malaria and for fight against the mixture of blood from different peoples, we were trained on how to differ many RDTs from different people by writing on RDT kit the name, age and village of the patient" (FGD1\&2 with CHWs).

\section{By taking concern with FGD 3 said that:}

"I experienced the case where child of my neighbor fell sick and used to take him to a traditional practitioner. But when I encouraged him to bring child to me for RDT; I treated him myself and found that he had malaria. I immediately gave him medicines and he recovered quickly. So, my neighbor learnt a lot from that and the one who is CHW 9 said that: whenever people bring me a sick person, I welcome him/ her warmly and treat him/her. This makes him/her feel satisfied and therefore raises the people's confidence in me. Furthermore, when I use RDT for malaria to help a sick person, I also take a sample of the blood of him/her to the health centre for verification. So, once microscopy confirms RDT results, I feel confident in carrying out my job" (FGD $1,2 \& 3)$.

The remaining KIIs were done to District community health supervisor and district health director where both confirm that majority of the CHWs are well experienced in the services they provide.

In KII 4 with district community health supervisor said that:

"CHWs are trained on RDT use. So, they use it well and give medicines to the sick person who is really in need of them, I mean the one who is suffering from malaria means that CHWs are fit for their job and master the use of RDT. We consider RDT positive results confirmed after laboratory tests by means of microscopy test. We also consider RDT negative results which are not confirmed. So, the comparison made shows that CHW know how to use RDT very well. All CHWs know that a sample of blood must last 72 hours. So, they bring the samples taken in not more than 3 days" (KII 4 ).

From the last KII with the district health director reported that: "Really, CHWs helps the sick person to know how to take medicines and invites him/her to take the first round of medicines in his presence. Then, the HCW tells the sick person that he/she should take the next round after a period of 8 hours and so on as the nurse would do. Additionally, CHWs do a follow-up and makes sure that all medicines are taken. The results of RDT and that of microscopy are always the same, we have never experienced in the past, when malaria becomes an epidemic in this area, we involved CHWs for a help. They came and spent time with us helping people who were suffer from malaria. They did it very well; they are experts if I may say so. HCWs are organized in a way that each $\mathrm{CHW}$ has a number of households to work with in village. CHWs regularly tours around those households sensitizing people and therefore creating or strengthening a friendship with them" (KII 5).

The above quotations show that CHWs are well experienced in the work they do including use of RDTs for malaria diagnosis services. This is in contrast to the study by Daniel et al. [17] done in Ghana, which showed that experience of community members and medicine outlet practitioners on the use of RDTs was low, and where they existed, were mainly limited to clinics and hospitals. However, both the community members and medicine outlet practitioners welcome the use of RDTs in detecting malaria cases at the community level in Ghana.

\section{Conclusion}

RDTs are the preferred method for the diagnosis of malaria by both communities and community health workers in Rubavu district and the community. Health workers have good experience on the use of rapid diagnostic test for malaria.

\section{Author's Contribution}

Gashegu Misbah: conceptualized the idea for the study, contributed in design and protocol preparation, performed analysis and interpretation of data and drafted the first manuscript.

Benon Asiimwe: contributed in the design and protocol preparation, involved in acquisition of data and participated in critical review of the subsequent draft of the manuscript.

Michael Habtu: provided assistance with the design, analysis and interpretation of data and participated in critical review of the subsequent draft of the manuscript. 
Citation: Gashegu M, Habtu M, Kansiime C, Mochama M, Asiimwe B (2017) Health Care Worker's Experience on Rapid Diagnostic Test (Rdt) for Malaria and Their Views on Community Acceptability of Rdt in Rubavu District, Rwanda: Qualitative Study. Malar Chemoth Cont Elimination 6: 157. doi: 10.4172/ 2470-6965.1000157

Page 4 of 6

Monica Mochama: assisted in design and protocol preparation.

Catherine Kansiime: assisted in design and protocol preparation, made a substantial contribution toward analysis and participated in critical review of the subsequent draft of the manuscript.

Each author has given final approval of the version to be published.

\section{Competing Interests}

The authors declare that they have no competing interests.

\section{Acknowledgement}

We would like to thank all community members, FGDs participants, data collectors and interviewers for the role they played in this study. We are also grateful to Professor MUNYANSHONGORE Cyprien of the University of Rwanda, school of public health for his valuable comments.

\section{References}

1. World Health Organisation (2010) World Malaria Report. Geneva: World Health Organisation.

2. World Health Organisation (2010) Guidelines for the treatment of malaria Second edition. Geneve: WHO Press.

3. Bradley EH, Webster TR, Baker D, Schlesinger M, Inouye SK, et al. (2004) Translating research into practice: speeding the adoption of innovative health care programs. Issue Brief Commonwealth Fund 1-12.

4. Karsh BT, Escoto KH, Beasley JW, Holden RJ (2006) Toward a theoretical approach to medical error reporting system research and design. Applied Ergonomics 37: 283-295.

5. Blandford A, Lunt P (2006) Co-evolving roles and technologies in the NHS: Barriers and forces for change ESRC E-Society Programme. E-Society Briefing 11.

6. Levy-Bruhl D, Soucat A, Osseni R, Ndiaye JM, Dieng B, et al. (1997) The Bamako Initiative in Benin and Guinea: improving the effectiveness of primary health care. Int J Health Plann Manage 12: 49-79.

7. Uzochukwu BSC, Onwujekwe E, Ezuma NN, Ezeoke OP, Ajuba MO, et al. (2011) Improving rational treatment of malaria: perceptions and influence of RDTs on prescribing behaviour of health workers in southeast Nigeria. PLoS One 2011
8. Ansah EK, Narh-Bana S, Epokor M, Akanpigbiam S, Quartey AA, et al (2010) Rapid testing for malaria in settings where microscopy is available and peripheral clinics where only presumptive treatment is available: a randomised controlled trial in Ghana. BMJ 2010; 340.

9. Skarbinski J, Ouma PO, Causer LM, Kariuki SK, Barnwell JW, et al. (2009) Effect of malaria rapid diagnostic tests on the management of uncomplicated malaria with artemether-lumefantrine in Kenya: a cluster randomized trial. Am J Trop Med Hyg 80: 919-926.

10. World Health Organization (2015) Guidelines for the treatment of malaria. $3^{\text {rd }}$ edition. Geneva, Switzerland WHO

11. Ajayi IO, Jegede AS, Falade CO, Sommerfeld J (2013) Assessing resources for implementing a community directed intervention (CDI) strategy in delivering multiple health interventions in urban poor communities in southwestern Nigeria: a qualitative study. Infect Dis Poverty 2: 1-25.

12. Gomes MF, Faiz MA, Gyapong JO, Warsame M, Agbenyega T, et al. (2009) Pre-referral rectal artesunate to prevent death and disability in severe malaria: a placebo-controlled trial. Lancet 373: 557-566.

13. Mukanga D, Tibenderana JK, Kiguli J, Pariyo GW, Waiswa P, et al. (2010) Community acceptability of use of rapid diagnostic tests for malaria by community health workers in Uganda. Malar J. 9:203.

14. Danquah DA, Buabeng KO, Asante KP, Mahama E, Plange CB, et al. (2016) Malaria case detection using rapid diagnostic test at the community level in Ghana: consumer perception and practitioners' experiences. Malaria Journal $15: 34$

15. Hamer DH, Brooks ET, Semrau K, Pilingana P, MacLeod WB, et al. (2012) Quality and safety of integrated community case management of malaria using rapid diagnostic tests and pneumonia by community health workers. Pathog Glob Health, 32-39.

16. Ajayi IO, Browne EN, Garshong B, Bateganya F, Yusuf B, et al. (2008) Feasibility and acceptability of artemisinin-based combination therapy for the home management of malaria in four African sites. Malar J. 7:6

17. Kilian AH, Tindyebwa D, Gulck T, Byamukama T, Rubaale T, et al. (2003) Attitude of women in western Uganda towards pre-packed, unit-dosed malaria treatment for children. Trop Med Int Health 431-438.

18. Danquah DA, Buabeng KO, Kwaku P. Asante KP, Mahama E, et al. (2016) Malaria case detection using rapid diagnostic test at the community level in Ghana: consumer perception and practitioners' experiences. Malaria Journal, 8:29. 